\title{
Density Functional Theory Study of Bone Tissues: the Role of Water in Conferring Bone Strength ${ }^{\dagger}$
}

\author{
Valerie Vaissier Welborn ${ }^{1, *}$ \\ 1 Department of Chemistry, Virginia Tech, Blacksburg, VA 24073 \\ * Correspondence: vwelborn@vt.edu; \\ $\uparrow$ Presented at Materials Chemistry and Physics (Materials Chemistry 2020) - International e-Conference
}

Received: 16.09.2020; Revised: 20.09.2020; Accepted: 24.09.2020; Published: 27.09.2020

\begin{abstract}
The unsurpassed mechanical properties of biomaterials stem from the intricate organization of inorganic and organic matter across length scales. In bone, water facilitates this organization, thereby playing an important structural role in addition to being a nutrient and waste transport medium. Water makes $10 \%$ of mammalian bone tissues and can be found in one of two states: bound (to the mineral phase) or mobile. While experimental studies were able to directly link the amount of bound water to bone strength, a molecular understanding of the interactions between the mineral (hydroxyapatite), organic (collagen) phase, and water is missing. In this talk, I will provide new insights into the water adsorption properties of bone tissues. I will present DFT calculations of water adsorption energy as a function of the environment, which includes an explicit solvent and human collagen fragments. I will show that the environment - rather than the mineral surface itself-governs the adsorption strength and mode. In particular, I will show that conditions consistent with aging tissues are associated with a lower density of adsorbed water molecules, which is a sign of weaker bones.
\end{abstract}

Keywords: DFT; water adsorption; inorganic-organic materials; biomaterials; explicit solvation; bound water; collagen.

(C) 2020 by the authors. This article is an open-access article distributed under the terms and conditions of the Creative Commons Attribution (CC BY) license (https://creativecommons.org/licenses/by/4.0/).

\section{Funding}

This research received no external funding.

\section{Acknowledgments}

The author thanks the Virginia Tech Chemistry Department Faculty Start-up for financial support and the Extreme Science and Engineering Discovery Environment (XSEDE) for allocating resources to this project.

\section{Conflicts of Interest}

The authors declare no conflict of interest. 